\title{
Natural Language Watermarking Based on Syntactic Displacement and Morphological Division
}

\author{
Mi-Young Kim, Osmar R. Zaiane, and Randy Goebel \\ Department of Computing Science, University of Alberta \\ Edmonton, AB, Canada \\ \{miyoung2,zaiane,goebel\}@cs.ualberta.ca
}

\begin{abstract}
This paper explores a method for Korean text watermarking based on a linguistic analysis scheme using morphemic and syntactic analysis. In this scheme, a predicate nominal is separated into its nominal and its predicate, and syntactic adverbial is displaced. Korean, as an agglutinative language, provides a good basis for this morpheme-based natural language watermarking because a word consists of several morphemes. A Korean word usually consists of a content morpheme and a function morpheme. However, a predicate nominal is an exception, having two content morphemes-nominal and predicate--and one function morpheme. So, we can divide a predicate nominal into a nominal and a predicate. In addition, we also perform syntax-based watermarking. We displace syntactic adverbials using the characteristic that most languages permit displacement of syntactic adverbials within its clause. Combining these morphemic and syntactic characteristics, we propose a method of language watermarking based on syntactic displacement and morphological division. To make our system more secure, we also include a sentence weight value and encode the weight value with a watermark bit. Our watermarking method doesn't change the meaning of the most marked sentences, and it also ensures the naturalness of the sentences.

From the experimental results, we show that the rate of unnatural sentences of marked text is reasonable, and the watermarking capacity is better than previous systems. The coverage of marked sentences is also reasonable. Experimental results also show that the marked text retains the same style, and also has the same information without semantic distortion.
\end{abstract}

\section{Introduction}

Text watermarking is an emerging technique in the intersection of natural language processing and the technologies of security. Text watermarking aims at embedding additional information in the text itself with the goals of subliminal encoding of information, of content and authorship authentication, and finally of enriching the text with metadata [2]. These kinds of watermarking techniques have been explored extensively for multimedia documents in the last decade[1]. In contrast, the studies on natural language watermarking are more recent, emerging in the last several years.

In $[3,4,6]$, the techniques of synonym substitution for watermarking have been addressed, and various attack scenarios have been described. In [8], Atallah et al. have attempted to use a so-called quadratic residues technique to insert a watermark within a given text via synonym substitution. The ambiguity induced on the word precision by the synonym substitution technique has led Topkara et al.[10] to consider an alternative syntax-based natural language watermarking. Their technique basically focuses on syntactic sentenceparaphrasing. In $[13,14]$, they propose morpheme segmentation and syntactic analysis for watermarking. However, their method has been shown to have relatively small information carrying ability.

Note that Korean, as an agglutinative language, differs significantly from Indo-European languages such as English, because one word consists of several morphemes. For this reason, we believe that Korean and other agglutinative languages provide a good basis for text watermarking based on division of a word by the characteristics of its morphemes. In addition, most languages permit the free order of a syntactic adverbial within its clause boundary. We exploit these two characteristics for text watermarking.

Korean words usually consist of a content morpheme and a function morpheme. However, 
predicate nominals are exceptional and typically consist of two content morphemes-nominal and predicate-together with one function morpheme. A predicate nominal is simply a predicate that is derived from a nominal. So, this paper proposes text watermarking by dividing a predicate nominal into these two components: a nominal and a predicate. We also try to transform a sentence by displacing syntactic adverbials. We embed this style of watermark in an original text, creating a ciphertext based on morphological division or syntactic displacement according to the choice of the watermark selector, which preserves the meaning. To make the system even more secure, we use a sentence weight and make the weight value carry a watermark bit.

\section{Previous Work}

M. Atallah et al. [7,8] proposed a technique for information hiding in natural language text. Moreover, they established the basic technique for embedding a resilient watermark in text by combining a number of information assurance and security techniques with the advanced methods and resources of natural language processing. A semantically-based scheme significantly improves the information-hiding capacity of English text by modifying the granularity of meaning of individual terms/sentences. However, this scheme is suitable only for English, and it was merely conceptual.

An alternative elaboration of this technique attempts to retain original text meaning, and proposes to replace words in the cover text with synonyms $[3,4,6]$. However, there is deterioration in documents in which importance is attached to any delicate semantic nuance when synonyms are substituted. There are also cases where incorrect words are selected as synonyms among many synonym candidate words. Moreover, the method requires a large synonymy dictionary and a huge collocation database [11].

There exist proposed methods for the text watermarking of agglutinative languages. H.M.Meral et al. [2] proposed morpho-syntactic tools for Turkish text watermarking, and O. Takizawa et al. [11] suggested the adjustment of new line positions for Japanese text. This method has the limitation that the message sender and recipient must share the same secret rule table, and the total rate of embedding is too low. M. Y. Kim [13] proposed morpheme segmentation for watermarking, but the embedding capacity was also too low.

Topkara et al. [10] also proposed a syntax-based natural language watermarking using syntactic sentence-paraphrasing. They insist that the syntactic approach is useful for natural language watermarking without semantic distortion. However, sentence paraphrasing can result in unnaturalness of the sentence.

M. Y. Kim [14] demonstrated a watermarking method based on syntactic analysis However, the embedding capacity was low. H. Wang et al. [12] proposed a watermarking method using a Chinese syntactic transformation. They introduce the notion of a sentence weight value for each sentence, and embed a watermark bit in the weight value.

Here, we propose a text watermarking method that combines morphological division and syntactic displacement, to improve coverage of watermarking. To improve system security, we assign a sentence weight for each sentence, and the watermark bit is hidden in the weight value.

We demonstrate that morpheme and syntax-based combined text watermarking can be effective. It improves the rate of embedding, and the coverage of the system better. It also helps retain the meaning and naturalness of a sentence. In the next section, we describe our method in detail.

\section{Text Watermarking based on Syntactic Displacement and Morphological Division}

\subsection{Embedding watermarking}

We propose two watermarking schemes: one is morphological division, and the other is syntactic displacement. A watermark selector phase chooses sentences that are applicable for watermarking, and then one of the two methods. If both of the methods can be applied to a sentence, we use a round-robin selection of the two methods to balance the result.

A quadratic residue function is adopted for watermarking [12]. An integer $q$ is called a quadratic residue modulo $\mathrm{z}$ if it is congruent to a perfect square $(\bmod z)$; i.e., if there exists an integer $y$ such that: $\mathrm{y}^{2}=\mathrm{q}(\bmod \mathrm{z})$; Otherwise, $\mathrm{q}$ is called a quadratic nonresidue $(\bmod \mathrm{z})$. $\mathrm{Z}$ should be a prime number where there are an equal number of residues and nonresidues. The quadratic residue function $\operatorname{qr}(k e y, W)$ is given in the following:

$\operatorname{qr}($ key, $W)=1$ if it is a quadratic residue $(\bmod k e y)$ 0 otherwise,

where key is a prime number and $W$ is a weight value.

We apply the weight value of Wang et al. [12] by exploiting the idea that the positions and lengths of words in a marked sentence are different from the 


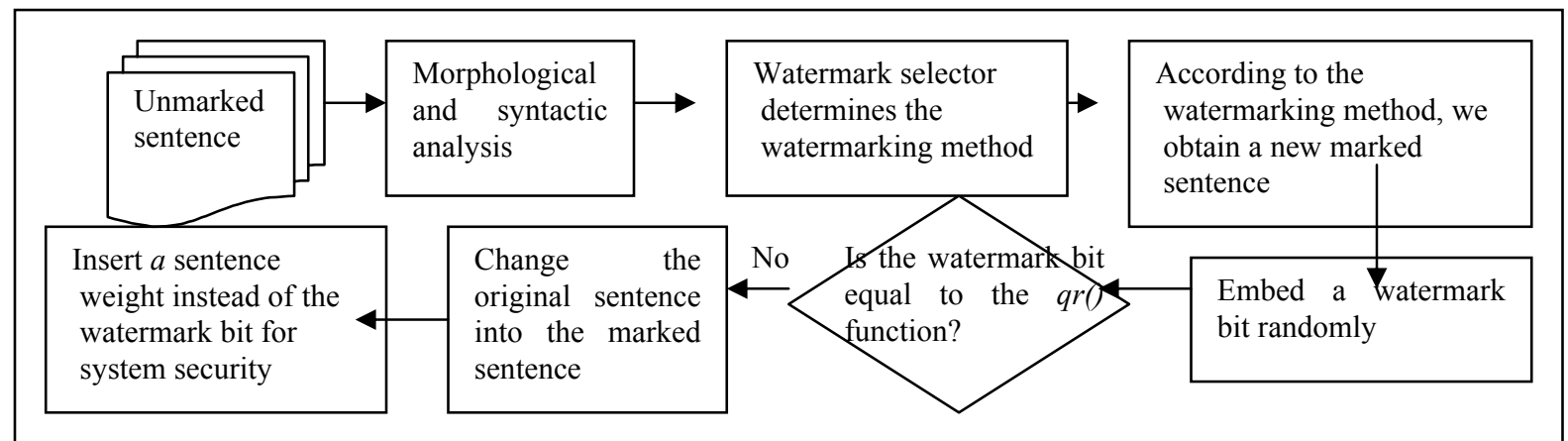

Fig. 1. Text watermarking procedure

\section{Chul-pan-doi-go : chul-pan (common noun) + doi (support predicate) + go (coordinate ending) \\ $\rightarrow$ Dividing it into two words}

(1) first new word : chul-pan (common noun)

(2) second new word : doi (support predicate) + go (coordinate ending)

$\rightarrow$ Inserting a functional word to the first word

(1) chul-pan (common noun) + i(subject case particle)

(2) doi (support predicate) + go (coordinate ending)

Original word: chul-pan-doi-go

Newly obtained words after morphological division : chul-pan-i doi-go

Fig. 2. Example of morphological division

original sentence. So, if we make a sentence weight based on the length of each word and its position, the weight value for the whole sentence will change if the sentence is changed. We can use the weight value $W$ of the original sentence, and a new value (either 1 or 0 ) for the sentence will be obtained through the $q r(k e y$, $W)$ function.

The embedding process of watermarking is as follows.

(1) Morphological and syntactic analyses are performed for all the sentences in the whole text, during which watermarking applicability is determined for each sentence.

(2) The weight value $\mathrm{Wj}$ for a sentence $\mathrm{Sj}$ is computed as follows.

Sentence weight $W$ for a sentence $\mathrm{S}$ is defined by:

$$
\mathrm{W}=\sum_{p=0}^{n} 2^{p} l(p)
$$

where $\mathrm{n}$ is the number of words in $\mathrm{S}$, and $l(\mathrm{p})$ is the length of $(\mathrm{p}+1)$ th word. Then, compare its quadratic residue function value with the watermarking bit $M i$. If they are equal, no change is made; otherwise, let the watermark selector choose the method for watermarking and obtain a marked sentence until $q r(k e y, W j)$ equals $M i$. If all transformations fail, no change is made to $W j$.

For example, in the sentence of Fig. 3, the $W$ value becomes

$2^{\wedge} 0 * 5+2^{\wedge} 1 * 1+2^{\wedge} 2 * 3+2^{\wedge} 3 * 3+2^{\wedge} 4 * 3=91$.

(3) Delete the above watermarking bit $M i$ from the watermark and insert $W j$ as used.

(4) Repeat steps (2)-(3) until the whole watermark information is embedded.

Fig. 1 shows the overall procedure of our text watermarking.

\subsection{Watermarking extraction}

We input the possibly marked sentence and obtain morphological and syntactic analysis results. The watermark extraction process is similar to the embedding process, except that we just calculate quadratic residue function value with the prime key with the sentence weights as the input arguments.

Then, we determine the watermarking method that must have been applied.

\subsection{Watermarking method: Morphological division}

As mentioned, because Korean is an agglutinative language, single word consists of several morphemes. 


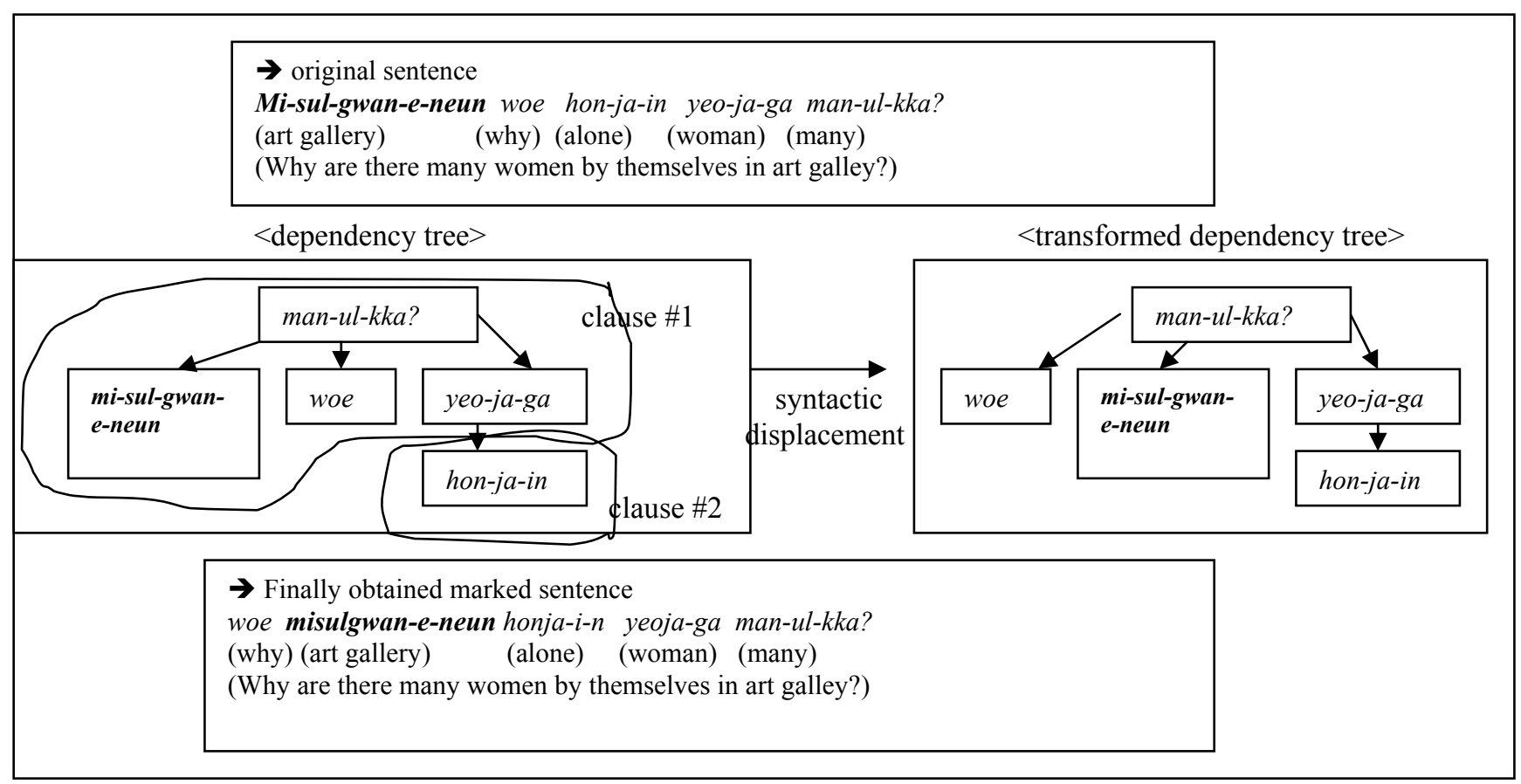

Fig. 3. Example of syntactic displacement

Typically, one word consists of a content morpheme and a function morpheme. However, some words exist that have more than one content morpheme. So, we try to find word types that have more than a content morpheme, and divide those words into two new words.

In Korean, a predicate nominal has two content morphemes - a nominal and a predicate. We choose the first predicate nominal in a sentence for division. We divide each predicate nominal into two new words, and insert a function morpheme for the first new word that does not have a function morpheme. An example is in Fig. 2.

A predicate nominal consists of "first content morpheme(nominal) + second content morpheme (support predicate) + function morpheme".

As shown in Fig. 2, the first new word consists of only a nominal, and the second new word consists of a predicate and a function morpheme - in Fig. 2, a coordinate ending is used as a function morpheme. Subsequently, the first new word does not have a function morpheme. So, we insert a relevant function morpheme based on the relationship between the nominal and predicate, determined as follows.

(1) If the support predicate indicates active voice (e.g. ' $h a$ ', 'siki'), then the nominal functions as the object of the predicate. So, we insert an object case particle--'eul' or 'reul'.
(2) If the support predicate indicates passive voice(e.g. 'doi'), then the nominal functions as the subject of the predicate. So, we insert a subject case particle -- ' $i$ ' or ' $g a$ '.

In Fig. 2, we can see that a subject case particle is inserted in the first new word because the support predicate('doi') exhibits a passive voice.

If a sentence can be changed based on morphological division, the watermark selector determines the sentence will be marked using morphological division, and the watermark bit and quadratic residue function value for the sentence is not equal, then we will get a marked sentence based on morphological division.

\subsection{Watermarking method: Syntactic displacement}

A syntactic dependency parser is used to determine the syntactic relation between words in a sentence. We use a Korean syntactic parser of M. Y. Kim et al. [5]. Fig. 3 shows an example of a syntactic dependency tree.

Although the Korean language admits relatively free word order, the boundaries over which a word can move is limited. We can displace a word within the clause that it belongs to. Adverbial, rather than other constituents, can move more freely in a sentence 
Table 1 Performances of our system

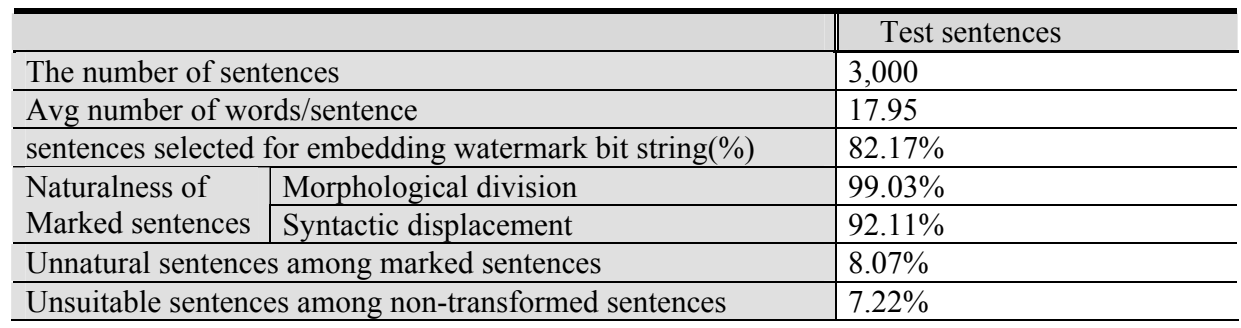

Table 2 Comparison of Performances with other systems

\begin{tabular}{l|l|l|l}
\hline & H. M. Meral[2] & H. Wang[12] & Our system \\
\hline Coverage of watermarking & & $11.44 \%$ & $82.17 \%$ \\
\hline information-hiding capacity & $0.81 \mathrm{bit} /$ sentence & $0.36 \mathrm{bit} / \mathrm{sentence}$ & $0.86 \mathrm{bit} /$ sentence \\
\hline
\end{tabular}

Table 3 Comparison of Performances for average edit

\begin{tabular}{l|l|l}
\hline & H. M. Meral[2] & Our system \\
\hline Edit-hit rate (\%) & $6.1 \%$ & $7.70 \%$ \\
\hline
\end{tabular}

without semantic distortion [9]. Therefore, as a target node for movement, we choose an adverbial constituent from a syntactic tree. We consider the displacement position from among the positions of the nodes at the same level hierarchy of the adverbial node in a clause.

Two kinds of displacement directions exist: left and right. In this study, we only consider moving to the right position. We choose the first syntactic adverbial that can be moved to the right position in a sentence, and move the adverbial to the right nearest position. In Fig. 3, 'mi-sul-gwan-e-neun' is an adverbial, and it moves to the right nearest position. Finally, from the modified syntactic tree, we generate a marked sentence as shown in the bottom of Fig. 3 .

If a sentence can be changed based on syntactic displacement, the watermark selector decides that the sentence will be marked using syntactic displacement, if the watermark bit and quadratic residue function value for the sentence are not equal, then we obtain a marked sentence using adverbial displacement.

\section{Experimental Results}

We have used the 3,000 declarative sentence set in the corpus of Matec99 (Morphological Analyzer and Tagger Evaluation Contest in Korean). As shown in Table 1, the average number of words/sentence is 17.95 .

We measure subjective rate by human as suggested by H.M.Meral et al. [2]. The evaluation method uses human evaluation of the text, and records their reactions by editing attempts. The subjects are given marked text and asked to edit them for improved intelligibility and style. This is a blind test because the subjects are not aware that text watermarking has taken place. Three humans have checked the sentences.

We measured the following performance characteristics.

1. Coverage of marked sentences

2. Naturalness of marked sentences

3. Human evaluation result

4. Information hiding capacity

We obtained the following results.

1. The coverage about the sentences selected for embedding watermark bit is $82.17 \%$.

2. Naturalness of morphological division is $99.03 \%$.

3. Naturalness of syntactic displacement is $92.11 \%$.

4. The embedding rate(information-hiding capacity) is $0.86 \mathrm{bit} /$ sentence.

5. The percentage of unnatural sentences among marked sentences is $8.07 \%$.

6. The percentage of unnatural sentences among non-transformed sentences is $7.22 \%$.

Table 1 shows the rate of unsuitable sentences among marked sentences and that among untransformed sentences. It is also interesting to note that sentences which have not transformed have also received edit hits at a rate of $8.07 \%$, implying that the edit hits between marked sentences and non-marked sentences are not so different. 
The average edit rate is $7.70 \%$, which shows worse result than that of H. M. Meral et al.[2] as shown in Table 3. However, the editing rate is very subjective, and we should also consider that the language and length of sentences are different between two methods.

In Table 2, the information-hiding capacity of our method is the best among the three methods.

We conclude that our natural language watermarking based on morpheme division and syntactic displacement shows reasonable performance without much semantic and stylistic distortion, and this method also shows good coverage. We also show improved watermarking capacity and we use sentence weight value to make our system more secure.

\section{Conclusion}

We propose natural language watermarking for Korean based on morphological division and syntactic displacement. By using the characteristics that a word in the agglutinative language usually consists of several morphemes, we divide the word that has two content morphemes into two new words, and a new function morpheme is inserted for the first new word that does not have a function morpheme. We also use the property that syntactic adverbials can be displaced within their clause boundaries. To improve watermarking capacity, we adopt a sentence weight value and make the weight carry a watermark bit. The experimental results show that the coverage of our method is $82.17 \%$, the average edit rate is $7.70 \%$, and the watermarking capacity is $0.86 \mathrm{bit} /$ sentence, outperforming previous systems.

We conclude that our watermarking method based on morpheme division, syntactic displacement, and the adoption of a sentence weight value is useful in watermarking of Korean text. Of course further experiments will confirm this further, and we will try to apply our method to other agglutinative languages to demonstrate that this method is effective on other languages.

\section{Acknowledgements}

This research was supported by the Alberta Ingenuity Centre for Machine Learning (AICML).

\section{References}

[1] I.Cox, M. L. Miller, J. A. Bloom, and M. Kaufman, "Digital Watermarking", 2002

[2] H. M. Meral, B. Sankur, A. S. Oszoy, T. Gungor, E. Sevinc "Natural language watermarking via morphosyntactic alterations", In Computer Speech and Language 23 pp. 107-125, 2009

[3] M. Topkara, C. M. Taskiran, E. J. Delp, "Natural language watermarking", SPIE Conf. On Security,
Steganography and Watermarking of Multimedia Contents, 2005

[4] C. M. Taskiran, M. Topkara, E. J. Delp, “Attacks on linguistic steganography systems using text analysis", SPIE Conf. On Security, Steganography and Watermarking of Multimedia Contents, pp. 313-336, 2006

[5] M. Y. Kim and J. H. Lee, "Two-Phase S-clause Segmentation", IEICE Transactions on Information and Systems, pp. 1724-1736, 2005

[6] U. Topkara, M. Topkara, M. J. Atallah, "The hiding Virtues of Ambiguity: Quantifiably Resilient Watermarking of Natural language Text through Synonym Substitutiions", In Proc. Of ACM Multimedia and Security Conference, 2006

[7] M. Atallah, V. Raskin, C. F. Hempelmann, M. Karahan, R. Sion, K. E. Triezenberg, U. Topkara, "Natural language watermarking and tamperproofing", Lecture Notes in Computer Sciences, 2002

[8] M. J. Atallah, V. Raskin, M. Crogan, C. Hempelmann, F. Kerschbaum, D. Mohamed, S. Naik. "Natural language watermarking: design, analysis, and proof-of-concept implementation", In Proc. Of the International Information Hiding Workshop, 2001

[9] J.I. Kwon, “The study of Korean grammar” Bak-I-Jeong, 1994

[10] M. Topkara, U. Topkara, M. J. Atallah, "Words are not enough: sentence level natural language watermarking", In Proc. of $4^{\text {th }} A C M$ International Proceedings of ACM Workshop on Content Protection and Security (in conjuction with ACM Multimedia), 2006

[11] Osamu Takizawa, Kyoko Makino, Tsutomu Matsumoto, Hiroshi Nakagawa, Ichiro Murase: Method of Hiding Information in Agglutinative Language Documents Using Adjustment to New Line Positions. KES (3) pp. 1039-1048, 2005

[12] H.Wang, X. Sun, Yu. Liu and Yo. Liu, "Natural Language Watermarking Using Chinese Syntatic Transformations", Information Technology Journal 7 (6): 904-910, 2008

[13] M. Y. Kim, "Natural language watermarking by morpheme segmentation", Proc. of the Asian Conference on Intelligent Information and Database Systems, pp.144-149, 2009

[14] M. Y. Kim "Text watermarking by syntactic analysis", Proc. of WSEAS International Conferences on Computers, pp.904-909, 2008 